\title{
AUTOPARALLEL DEVIATION IN THE GEOMETRY OF LYRA
}

\author{
J.R. Vanstone \\ (received June 17, 1960)
}

1. Introduction. One of the fruitful tools for examining the properties of a Riemannian manifold is the study of "geodesic deviation". The manner in which a vector, representing the displacement between points on two neighbouring geodesics, behaves gives an indication of the difference between the manifold and an Euclidean space. The study is essentially a geometrical approach to the second variation of the lengthintegral in the calculus of variations [1]. Similar considerations apply in the geometry of Lyra [2] but as we shall see, appropriate analytical modifications must be made. The approach given here is modelled after that of Rund [3] which was originally designed to deal with a Finsler manifold but which applies equally well to the present case.

We shall use the notation of Scheibe [4] as far as possible except that our curvature tensor $\mathrm{K}_{r}{ }^{i} \mathrm{hk}$ is the negative of his. This convention conforms more closely with the classical usage.

2. Fundamental definitions. In the geometry of Lyra the autoparallels are the curves $x^{i}(t)$ whose tangent vectors $\xi i=x^{\circ} \dot{x}(t)$ satisfy the differential equations

$$
d \xi i / d t+\tilde{r} j_{j k}^{i} \xi^{j} \xi^{k}=0,
$$

where

$$
\tilde{\Gamma}_{j k}^{i}=\Gamma_{j k}^{i}-\frac{1}{2} \delta_{j}^{i} \phi_{k}, \Gamma_{j k}^{i}=\Gamma_{k j}^{i} .
$$

The significance of the functions involved here is fully explained in Lyra, or in the preceding paper of Sen in this journal.

Corresponding to the connection (2) we may define a covariant derivative

$$
x_{; j}^{i}=\left(x^{0}\right)^{-1} x_{, j}^{i}+\tilde{\Gamma}_{r}^{i} x^{r} ; x_{, j}^{i}=\partial x^{i} / \partial x^{j},
$$

Canad. Math. Bull. vol. 3, no. 3, September 1960 
where $X^{i}$ is an arbitrary vector (in the sense of Lyra). By demanding that the product rule remain valid, this derivative can be defined for arbitrary tensors. In particular, if $\phi$ is a scalar, we have $\phi_{; \mathrm{k}}=\left(\mathrm{x}^{0}\right)^{-1} \phi_{, \mathrm{k}}$. Furthermore if a symmetric metric tensor, $g_{i j}$, is defined on the space, so that we interpret

$$
F(x, x) \equiv\left(g_{i j}(x) x^{i} x^{j}\right)^{\frac{1}{2}}
$$

as the length of a vector $X^{i}$, we have

$$
g_{i j ; k}=\left(x^{0}\right)^{-1} g_{i j, k}-g_{r j} \tilde{\Gamma}_{i k}^{r}-g_{i r} \tilde{\Gamma}_{j k}^{r} \text {, }
$$

and the condition that the space be metric is

$$
g_{i j ; k}=0 \text {. }
$$

This condition, together with (2), yields [2]

$$
\Gamma_{j k}^{i}=\left(x^{0}\right)^{-1}\left\{\begin{array}{c}
i \\
j k
\end{array}\right\}+\frac{1}{2}\left(\delta_{j}^{i} \phi_{k}+\delta_{k}^{i} \phi_{j}-g_{j k} \phi^{i}\right) \text {. }
$$

We use $g_{i j}$ and its inverse $g^{i j}=\left[\operatorname{det}\left(g_{i j}\right)\right]^{-1}$ (cofactor $g_{i j}$ ) to raise and lower tensor indices.

The commutation formulae corresponding to (3) are

$$
\begin{aligned}
& \mathrm{X}_{; \mathrm{i} ; \mathrm{k}}^{\mathrm{i}}-\mathrm{X}^{\mathrm{i}} ; \mathrm{k} ; \mathrm{h}=-\mathrm{X}^{\mathrm{i}} ; \mathrm{r}^{\mathrm{H}}{ }_{h k}+\mathrm{X}^{\mathrm{r}} \mathrm{K}_{\mathrm{r}}{ }_{\mathrm{h} k}^{\mathrm{i}} \text {, } \\
& X_{i ; h ; k}-X_{i ; k ; h}=-X_{i ; r} H_{h k}^{r}-X_{r} K_{i h k}^{r} \text {, }
\end{aligned}
$$

where

$$
\begin{aligned}
H_{h k}^{r}= & \frac{1}{2}\left[\delta_{h}^{r}\left(\dot{\phi}_{k}-\phi_{k}\right)-\delta_{k}^{r}\left(\dot{\phi}_{h}-\phi_{h}\right)\right], \\
\dot{\phi}_{h}= & \left(x^{0}\right)^{-1}\left[\ln \left(x^{0}\right)^{2}\right]_{h^{\prime}} \\
K_{r h k}^{i}= & \left(x^{0}\right)^{-2}\left[\left(x^{\circ} \tilde{\Gamma}_{r h}^{i}\right)_{k}-\left(x^{\circ} \tilde{\Gamma}_{r k}^{i}\right), h\right. \\
& \left.+\left(x^{\circ} \tilde{\Gamma}_{r h}^{s}\right)\left(x^{\circ} \tilde{\Gamma}_{s k}^{i}\right)-\left(x^{\circ} \tilde{\Gamma}_{r k}^{s}\right)\left(x^{\circ} \tilde{\Gamma}_{s h}^{i}\right)\right] .
\end{aligned}
$$

The formulae (7) may be extended to tensors of higher order. 
In particular,

$$
g_{i j ; h ; k}-g_{i j ; k ; h}=-g_{i j ; r} H_{h k}^{r}-g_{r j} K_{i}^{r}{ }_{h k}-g_{i r} K_{j h k}^{r} \cdot
$$

In view of $\left(5^{\prime}\right)$, then, it follows that

$$
\mathrm{K}_{\mathrm{ijhk}}+\mathrm{K}_{\mathrm{jihk}}=0
$$

and hence $\mathrm{K}_{\mathrm{ijhk}}$ is skew-symmetric in its first two and last two indices.

The identity, where $\mathrm{x}^{\mathrm{k}}=\mathrm{x}^{\mathrm{k}}(\mathrm{x})$,

$$
\left(x^{0}\right)^{-1} F(x, X), k-\partial F(x, X) / \partial x^{r} \tilde{\Gamma}_{j k}^{r} x^{j}=0
$$

will also be useful in the sequel. This is obtained by differentiating (4) convariantly with respect to $x^{k}$. Since $F(x, X)$ is a scalar, the left side yields $\left(x^{0}\right)^{-1} \quad\left[F, k+\left(\partial F / \partial X^{r}\right) X^{r}, k\right]$ and, in view of $\left(5^{\prime}\right)$, the right side gives $(F)^{-1} g_{i j} X^{i} X^{j} ; k^{\prime}$ Using (3) and noting that $\partial F / \partial X^{j}=(F)^{-1} g_{i j} X^{i}$, we obtain (11). It is noteworthy that the partial derivatives of $\mathrm{X}^{\mathrm{k}}$ do not occur in (11).

3. The general problem. We consider a two-dimensional subspace $\mathrm{L}_{2}$ which may be represented parametrically by $x^{i}=x^{i}(u, v)$. The tangent vectors (in the sense of Lyra) to the coordinate curves on this surface are

$$
\xi^{i}=x^{\circ} \partial x^{i} / \partial u=x^{\circ} x_{u}^{i} ; \eta^{i}=x^{\circ} \partial x^{i} / \partial v=x^{\circ} x_{v}^{i}
$$

and we assume that the se are linearly independent throughout the region under consideration. Note that

$$
\xi_{v}^{i}-\eta_{u}^{i}=\frac{1}{2}\left(\delta i_{n}^{i} \phi_{m}-\delta_{m}^{i} \dot{\phi}_{n}\right) \xi^{n} \eta^{m},
$$

by virtue of the definition (8) of $\oint_{\mathrm{m}}$. defined by

$$
\delta x^{i} / \delta u=x^{i} ; m \xi^{m} ; \delta x^{i} / \delta v=x^{i} ; m \eta^{m}
$$

In particular,

$$
\delta \xi^{i} / \delta v=\left[\left(x^{0}\right)^{-1} \xi^{i}, m+\xi^{r} \tilde{\Gamma}_{r m}^{i}\right] x^{o} x_{v}^{m}=\xi_{v}^{i}+\tilde{\Gamma}_{r m}^{i} \xi^{r} \eta^{m} .
$$


Carrying out a similar calculation for $\delta \eta^{i} / \delta u$ and taking the difference, we find, by $\left(12^{\prime}\right),(2)$ and $(8)$, that

$$
\delta \xi^{i} / \delta v-\delta \eta^{i} / \delta u=H_{n m}^{i} \xi^{n} \eta^{m} .
$$

The mixed derivatives $\delta^{2} \mathrm{X}^{\mathrm{i}} / \delta_{\mathrm{v}} \delta_{\mathrm{u}}$ and $\delta^{2} \mathrm{X}^{\mathrm{i}} / \delta_{\mathrm{u}} \delta_{\mathrm{V}}$ are easily calculated from (13). It follows that

$$
\begin{aligned}
& \delta^{2} x^{i} / \delta v \delta u-\delta^{2} x^{i} / \delta u \delta v \\
= & \left(x^{i} ; n ; m-x^{i} ; m ; n\right) \xi^{n} \eta^{m}+x_{; n}^{i}\left(\delta \xi^{n} / \delta v-\delta \eta n / \delta u\right) \\
= & x^{r_{1}}{ }_{r m m}^{i} \xi^{n} \eta^{m},
\end{aligned}
$$

where the second equality is obtained from (7) and (14). In particular

$$
\delta^{2} \xi^{\mathrm{i}} / \delta \mathrm{v} \delta \mathrm{u}-\delta^{2} \xi^{\mathrm{i}} / \delta \mathrm{u} \delta \mathrm{v}=\mathrm{K}_{\mathrm{r} n \mathrm{~nm}}^{\mathrm{i}} \xi^{\mathrm{r}} \xi^{\mathrm{n}} \eta^{\mathrm{m}} .
$$

We are now in a position to examine the deviation of neighbouring curves $\mathrm{v}=$ constant. Let $\mathrm{c}$ be an arbitrary finite constant and let $\varepsilon$ be a small constant. Consider the curves $C: v=c$ and $C *: v=c+\varepsilon$. Since the curves $u=$ constant intersect both $C$ and $C *$, a natural correspondence is established between points of these two curves. We shall find it convenient to consider a more general correspondence. Assuming that a function $f(u)$ is given, which has a continuous second derivative and which is of the same order of magnitude as $\varepsilon$, we let the point $A\left(x^{i}\right)=A(u)$ on $C$ correspond to the point $B\left(u^{*}\right)$ on $C^{*}$, where $u^{*}=u+f(u)$. The vector $z^{i}$ associated with the displacement from $A$ to $B$ is

$$
z^{i}=x^{\circ} d x^{i}=f(u) \xi^{i}+\varepsilon \eta^{i} \text {, }
$$

neglecting quantities of the order of $\varepsilon^{2}$.

The problem, then, is to ascertain the behaviour of $z^{i}$ as $A(x)$ moves along $C$. This is accomplished by formulating a set of differential equations which characterize the components of $z^{i}$. In order that this system be independent of the reference system, it must be tensorial and hence the covariant derivatives defined above, instead of ordinary derivatives, are used.

If we differentiate (16) covariantly with respect to $u$, taking (14), (16) and the skew-symmetry of $\mathrm{H}^{\mathrm{i}} \mathrm{nm}$ into account, 
we obtain

$$
\delta z^{i} / \delta u=\delta\left(f \xi^{i}\right) / \delta u+\varepsilon \delta \xi^{i} / \delta v-H_{n m}^{i} \xi^{n} z^{m} .
$$

A second differentiation will give rise to a term $\varepsilon \delta 2 \xi i / \delta \mathrm{u} \delta \mathrm{v}$ in the right-hand side. This may be replaced by $\varepsilon \delta^{2} \xi i / \delta v \delta u-K_{r}^{i} n m \xi^{r} \xi^{n} z^{m}$ in view of (15'), (16) and the skew-symmetry of $K_{r} i n m$ in $n$ and $m$. Rearranging terms we therefore have

$$
\begin{aligned}
& \delta^{2} z^{i} / \delta u^{2}+\left(H^{i}{ }_{n m}^{n}\right) \delta z^{m} / \delta u \\
& +\left[K_{r}^{i} n m \xi^{r} \xi^{n}+\delta\left(H_{n m}^{i} \xi^{n}\right) / \delta u\right] z^{m} \\
& =\varepsilon \delta^{2} \xi^{i} / \delta v \delta u+\delta^{2}\left(f \xi^{i}\right) / \delta u^{2} .
\end{aligned}
$$

This is the most general deviation equation. Both $f(u)$ and the coordinate curves are of a general nature.

4. Autoparallel deviation. The most interesting case of equation (18) occurs when the curves $v=$ constant are autoparallels and the parameter $u$ is taken to be $s$, the arc-length of these curves. The latter assumption means that $\xi^{i}$ is a unit vector field, since

i. e.

$$
d s^{2}=\left[g_{i j}\left(x^{\circ} d x^{i}\right)\left(x^{\circ} d x^{j}\right)\right]^{\frac{1}{2}}
$$

$$
F(x, \xi)=\left(g_{i j} \xi^{i} \xi^{j}\right)^{\frac{1}{2}} \equiv 1 \text {. }
$$

In view of (1) (with $t=s$ ) and (13) (with $X^{i}=\xi^{i}, u=s$ ), the former assumption is equivalent to

$$
\delta \xi^{i} / \delta s=0 .
$$

Since this condition is independent of the value of $v$, we also have $(\delta \xi i / \delta s)_{\mathrm{v}}=0$ and since

$$
\delta^{2} \xi^{i} / \delta v \delta s \equiv\left(\delta \xi^{i} / \delta s\right)_{v}+\tilde{r}_{r m}^{i}\left(\delta \xi^{r} / \delta s\right) \eta^{m},
$$

it follows that $\delta^{2} \xi i / \delta \vee \delta s=0$. After these simplifications equation (18) becomes

$$
\begin{aligned}
& \delta^{2} z^{i} / \delta s^{2}+\left(H_{n m}^{i} \xi^{n}\right) \delta z^{m} / \delta s \\
& +\left[K_{r}^{i} n m \xi^{r} \xi^{n}+\delta\left(H_{n m}^{i} \xi^{n}\right) / \delta s\right] z^{m} \\
& =f^{\prime \prime}(s) \xi^{i} \text {, }
\end{aligned}
$$


which is the equation of autoparallel deviation.

This equation is more complicated than the corresponding Riemannian analogue [1] where the function $f$ is usually taken to be zero and, more essentially, the tensor $\mathrm{H}^{\mathrm{i}} \mathrm{nm} \xi^{\mathrm{n}}$ does not occur so that the first derivatives of $z^{i}$ do not appear.

As Sen has pointed out, elsewhere in this journal, the necessary and sufficient condition that the class of geodesics of the space (i.e. the solutions of the problem $\delta \int \mathrm{ds}=0$ in the calculus of variations) coincides with the class of autoparallels, is $\phi_{\mathrm{k}}=\phi_{\mathrm{k}}$. This condition implies that $\mathrm{H}^{\mathrm{r}} \mathrm{hk}$ vanishes, by (8). Conversely if $\mathrm{H}^{\mathrm{r}}$ hk vanishes then so does $\mathrm{H}^{\mathrm{r}}{ }_{\mathrm{rk}}=\frac{1}{2}(\mathrm{n}-1)\left(\dot{\phi}_{\mathrm{k}}-\phi_{\mathrm{k}}\right)$ and hence the condition of Sen is fulfilled. Such spaces are therefore characterized by. $\mathrm{H}^{\mathrm{r}} \mathrm{hk}=0$ and in this case (20) becomes

$$
\delta^{2} z^{i} / \delta s^{2}+K_{r n m}^{i} \xi^{r} \xi^{n} z^{m}=f^{\prime \prime}(s) \xi^{i} \text {. }
$$

5. A first integral. The geometrical structure of the situation described above is such that equation (20) admits an easily derived first integral. We put $\xi_{i}=g_{i j} \xi^{j}$ and note that,

$$
\xi_{i} \xi^{i}=1 ; \quad \delta \xi_{i} / \delta s=0,
$$

by virtue of (19) and (5'). Thus, by (10), inner multiplication of $(20)$ with $\xi$ i yields

$$
\delta\left[\delta\left(\xi_{i} z^{i}\right) / \delta s+H_{n m}^{i} \xi_{i} \xi^{n_{z}^{m}}-f^{\prime}(s)\right] / \delta s=0 .
$$

The constant inside the square brackets here may be shown to be zero in the case under consideration. More generally, we prove the

LEMMA. If a two-dimensional subspace $L_{2}$, together with $\xi^{i}$ and $\eta^{i}$ are defined as in $\xi 3$ and if $u$ is chosen to be the arc-length of the arbitrary_curves $v=$ constant, then

$$
\left(\partial F / \partial \xi^{i}\right)\left(\delta \xi^{i} / \delta u\right)=0 ;\left(\partial F / \partial \xi^{i}\right)\left(\delta \xi^{i} / \delta v\right)=0 \text {. }
$$

Proof. Since $\xi^{i}$ is an unit field, equation (19) holds throughout $L_{2}$. Hence $F_{i}, i d x^{i}+(\partial F / \partial \xi i) d \xi 1$, where $d x^{i}=\left(x^{0}\right)^{-1}\left(\xi i d u+\eta{ }^{i} d v\right)$ and $d \xi^{i}=\left(\partial \xi^{i} / \partial u\right) d u+\left(\partial \xi^{i} / \partial v\right) d v$ and $(d u, d v)$ is an arbitrary displacement, vanishes. Equating the coefficients of $d u$ and $d v$ separately to zero and sub- 
stituting for $F$, from equations (11), we obtain the relations (23). This result does not involve the assumption that the curves $v=$ constant are autoparallels.

If we now assume that $\mathrm{u}=\mathrm{s}$ in (17) and use (23), it follows that

$$
\xi_{i}\left(\delta z^{i} / \delta s\right)=f^{\prime}-H^{i} n m \xi_{i} \xi^{n} z^{m} \text {, }
$$

since $\partial F / \partial \xi^{i}=\xi_{i}$ when $F(x, \xi) \equiv 1$. Thus (24) represents a first integral of (18) if $u=s$. In particular, when we are dealing with autoparallel deviation so that the second equation of (21) holds, we obtain the result stated above, namely that

$$
\left[\delta\left(\xi_{i} z^{i}\right) / \delta s\right]+H^{i} n m \xi_{i} \xi^{n} z^{m}=f^{\prime}(s)
$$

is a first integral of (20). In the case when geodesics coincide with autoparallels this reduces further to $d\left(\xi_{i} z^{i}-f\right) / d s=0$ (the derivative $\delta / \delta s$ applied to a scalar is equivalent to $d / d s$ ) and hence $f$ may be interpreted to within an additive constant as the component of $z^{i}$ along $C$. If, as well, we assume that $\mathrm{f}=0$, it follows that this component is constant along $C$. In general we say that the deviation is normal if this component is zero (i.e. $\xi_{i} z^{i}=0$ ) and equation (25) must then be taken as the definition of $f$.

6. The two-dimensional case. We assume that the space under consideration is two-dimensional and we define $z^{i}$ to be the unit vector in the direction of $z^{i}$, which we assume to be a normal deviation. Thus

$$
g_{i j} z^{i} z^{j}=z_{i} z^{i}=1 ; \xi_{i} z^{i}=0
$$

It follows that

$$
z_{i}\left(\delta z^{i} / \delta s\right)=0 ; \xi_{i}\left(\delta z^{i} / \delta s\right)=0,
$$

by (21). Since $Z^{i}$ may not be co-directional with $\xi_{i}$ and since the space is two-dimensional, we conclude from (27), that

$$
\delta z^{i} / \delta s=0
$$

There exists a scalar $z$ such that $z^{i}=z z^{i}$. If this is substituted in (20) and an inner multiplication with $\mathrm{Z}_{j}$ carried out, the result is 


$$
z^{\prime \prime}+\alpha z^{\prime}+\left(K+\alpha^{\prime}\right) z=0
$$

where

$$
\alpha=H^{i}{ }_{n m} Z_{i} \xi^{n} Z^{m}, K=K_{r}^{i} \xi^{r} z_{i} \xi^{n} z^{m},
$$

and where (26) and (28) have been taken into account.

$T$ his is a scalar equation of deviation with the same significance as (20) since the vector $z^{i}$ only becomes null when $z=0$. The first derivative may be eliminated by the substitution $z=\zeta \exp \left(-\frac{1}{2} \int^{s} \alpha(\sigma) d \sigma\right)$. The result is

$$
\zeta^{\prime \prime}+\left[K+\frac{1}{2} \alpha^{\prime}-\frac{1}{4} \alpha^{2}\right] \zeta=0 \text {. }
$$

The zeros of $\zeta$ are the zeros of $z$. Successive zeros of $\zeta$ are points where neighbouring geodesics coincide. In the language of the calculus of variations, they are conjugate points. Equation (31) has the same form as the analogue for Riemannian spaces except that the scalar of curvature is modified by the addition of $\frac{1}{2} \alpha^{\prime}-\frac{1}{4} \alpha^{2}$. The analysis of the space proceeds in the same manner.

In conclusion we remark that a similar reduction of equations $(20)$ to a single scalar equation is possible in a space of any number of dimensions. The coefficients are somewhat more complicated because the relation (28) will not, in general, hold. However, an equation of the type (31) will arise in the same manner as above.

Estimates of the separation of zeros of $\zeta$ may be deduced from bound s on the coefficient $K+\frac{1}{2} \alpha^{\prime}-\frac{1}{4} \alpha^{2}$ after a theorem of Sturm proved in Blaschke [1]. In fact Sturm proved that if the coefficient in equations of the type (31) is increased in magnitude, successive zeros of the solution of the new equation will be separated by successive zeros of the solution of the original equation. In particular, then, if $K+\frac{1}{2} \alpha^{\prime}-\frac{1}{4} \alpha^{2} \geqslant A-2$, where $A$ is a positive constant, we may consider

$$
\eta^{\prime \prime}+A^{-2} \eta=0 \text {. }
$$

whose solution has successive zeros a distance $\mathrm{s}=\pi \mathrm{A}$ apart. It follows from the theorem of Sturm that successive zeros of $\zeta$ are no more than $\pi$ A apart. 


\section{REFERENCES}

1. W. Blaschke, Differential Geometry,(New York, 1945) p. 212 , et seq.

2. G. Lyra, Über eine Modifikation der riemannschen Geometrie; Math. Z. 54 (1951),52-64.

3. H. Rund, The Differential Geometry of Finsler Spaces, Springer-Verlag (Berlin-Gottingen-Heidelberg, 1959), 111-119.

4. E. Scheibe, Ưber einen verallgemeinerten affinen Zusammenhang, Math. Z. 57 (1952), 65-74.

University of Toronto 